\title{
Eficacia de la ivermectina para el control de nematodos gastrointestinales en burros (Equus asinus) en el altiplano mexicano
}

\author{
Guadalupe Galicia-Velázquez ${ }^{\text {a }}$ \\ Arturo Villarreal-Nieto ${ }^{\text {a }}$ \\ Cristina Guerrero-Molina ${ }^{\text {a }}$ \\ Cintli Martínez-Ortiz-de-Montellano a*
}

\begin{abstract}
${ }^{a}$ Universidad Nacional Autónoma de México, Facultad de Medicina Veterinaria y Zootecnia, Circuito Exterior, Ciudad Universitaria, Av. Universidad 3000. 04510, Ciudad de México, México.
\end{abstract}

*Autor de correspondencia: cintli@unam.mx

\section{Resumen:}

El burro se ha empleado como animal de trabajo durante siglos y el $96 \%$ de la población mundial se encuentra en países en desarrollo. Los nematodos gastrointestinales con resistencia antihelmíntica (RAH) son el problema parasitario más grave de los équidos. Este estudio analiza el fenómeno de RAH a la ivermectina (IVM) en burros, y se consideran umbrales económicos con la evaluación de las prácticas por parte de los propietarios a través de encuestas. Con 53 burros del Altiplano Mexicano, el experimento se dividió en dos etapas: 1) en 53 animales se determinaron umbrales económicos y se formaron los grupos experimentales. 2) se realizó la prueba de la eficacia de la IVM y se establecieron dos grupos experimentales $(n=30)$, grupo tratado y grupo testigo sin tratamiento. El umbral económico de huevos por gramo de heces fue de 600 y el umbral de la condición corporal (CC) del $91 \%$ de los animales fue aceptable (2.5 a 3.5). A mayor CC, la descarga de huevos obtenida fue menor $(P<0.05)$ de las 100 larvas identificadas, el $63 \%$ fueron ciatostómidos, el resto fueron grandes estrongílidos. En esta población de nematodos, la eficacia de la IVM fue del $100 \%$. Con las encuestas, el $80 \%$ de los propietarios admite utilizar como única estrategia 
el servicio de los médicos veterinarios zootecnistas voluntarios, el cual consiste en IVM al $1 \%$. La IVM es un recurso todavía valioso y debe utilizarse adecuadamente para evitar RAH. Este es el primer paso para la desparasitación selectiva dirigida en équidos en México.

Palabras clave: Burros, Equus asinus, Ciatostómidos, Nematodos, Ivermectina, Resistencia antihelmíntica.

Recibido: 05/10/2018

Aceptado: 05/04/2019

\section{Introducción}

El burro (Equus asinus) se ha empleado como animal de trabajo durante 5,000 años ${ }^{(1)}$ y más del $96 \%$ de la población mundial se encuentra en países en desarrollo. En México, se calcula que existen alrededor de 3.3 millones de burros ${ }^{(2)}$. En el altiplano mexicano, estos son mayoritariamente utilizados para las actividades agrícolas ${ }^{(2)}$. En algunos casos estos burros sufren de una deficiente nutrición, ya que son alimentados con esquilmos agrícolas y suplementados con granos de baja calidad o concentrados comerciales ${ }^{(1-2)}$. A su vez, el burro es hospedador de un gran número de parásitos y los ciclos de vida son similares a los presentes en caballos, por lo que estos pueden actuar como un significativo reservorio para la infección de otros équidos ${ }^{(3)}$. Los parásitos de mayor importancia en el caso de los équidos, son algunos helmintos como Anoplocephala perfoliata o Parascaris equorum, pero los que tienen un mayor impacto debido a las implicaciones clínicas de la migración larvaria e hipobiosis que presentan ${ }^{(4,5)}$, son los de la familia Strongylida, donde se ubica a los nematodos gastrointestinales (NGI) de la subfamilia Cyathostominae, también conocidos como pequeños estrongílidos, y los de la subfamilia Strongylidae (Strongylus spp), conocidos como grandes estrongílidos ${ }^{(6,7)}$. Estas nematodosis gastrointestinales en burros son quizás uno de los más grandes retos en el manejo clínico, ya que los burros que presentan cargas significativamente altas de helmintos pueden estar aparentemente sanos y rara vez observarse los signos clínicos en comparación con los caballos ${ }^{(8)}$.

Se sabe que, entre caballos y burros, existen grandes diferencias en comportamiento y fisiología, y específicamente en el mecanismo por el cual metabolizan ciertas drogas y a su vez, responden de manera distinta a las patologías que los afectan ${ }^{(9)}$. Esto no se ve reflejado en la industria farmacéutica, ya que los burros tienen un impacto económico limitado comparado con los caballos ${ }^{(9)}$. Por lo tanto, debido a la subdosificación, existe la posibilidad de encontrar burros con NGI resistentes a los químicos, como se ha demostrado en otras especies domésticas ${ }^{(10,11)}$. En los animales domésticos de todo el mundo, los NGI y su 
resistencia antihelmíntica (RAH), representan problemas de salud, económicos y productivos $^{(12,13)}$. El fenómeno de la RAH es ampliamente estudiado en México y América Latina sobre todo en rumiantes ${ }^{(12-14)}$ y otros équidos ${ }^{(5)}$. Existen algunos estudios en algunas zonas ecológicas del altiplano y centro del país, donde se mide el efecto antiparasitario de la ivermectina (IVM) en équidos ${ }^{(15)}$, pero nunca ha sido medida la eficacia en burros. En la región del Altiplano Mexicano, los médicos veterinarios en campo, desde hace más de 10 años proporcionan gratuitamente la desparasitación a burros en al menos dos veces al año con IVM al 1\% oral (comunicación personal Prado-Ortiz, O.), por lo que el desarrollo de la RAH de la población parásita es inminente. Debido a estas prácticas es relevante la realización de estudios que revelen el panorama actual de la RAH en la región.

Actualmente, cuando se quiere enfrentar la RAH, antes que nada, deben conocerse aspectos importantes de la epidemiología de la enfermedad de manera regional, local y particular ${ }^{(11,16,17)}$. Para esto, el establecimiento de criterios propios de umbrales económicos como la distribución de la eliminación de huevos por gramo de heces (HPG), los valores de la condición corporal (CC) y la identificación de géneros de nematodos existentes en la zona ecológica a estudiar, son necesarios, así como la detección de posibles prácticas que indiquen fallas en el tratamiento y causen la $\mathrm{RAH}^{(18,19)}$. En este estudio se analiza el fenómeno de RAH en burros y se consideran estos umbrales con la evaluación de las prácticas del propietario. Esto contribuye a la planeación de las distintas estrategias para retrasar la RAH y prolongar la efectividad de un fármaco, con miras en un futuro de lograr un Control Integrado Parasitario (CIP) en burros.

\section{Material y métodos}

El estudio fue realizado en cuatro comunidades de los municipios de Tecozautla, Hidalgo (Aljibes y San Pedro) y San Juan del Río, Querétaro (Santa Rosa Xajay y Vaquerías), pertenecientes al altiplano mexicano.

\section{Animales}

Se eligieron burros de estas comunidades mencionadas, porque reciben continuamente apoyo de médicos veterinarios zootecnistas (MVZ) voluntarios, los cuales proporcionaron información de los propietarios de burros y datos de protocolos de tratamiento de estos. El último tratamiento antihelmíntico aplicado fue con IVM al $1 \%$ vía oral, seis meses previos al experimento. Cabe mencionar que los burros de estas comunidades llevan más de 10 años siendo atendidos por estos médicos y ellos nunca han utilizado otra familia de antihelmínticos ni fármacos. 


\section{Diseño experimental}

Se seleccionaron 53 burros (Equus asinus), sin diferencia de sexo, dentro de un rango de edad de 3 meses hasta 25 años. El experimento se dividió en dos etapas. En la $1^{a}$ etapa, los 53 animales permitieron la determinación de los umbrales económicos (explicados más adelante) en la zona ecológica y la formación de los grupos experimentales de la $2^{\mathrm{a}}$ etapa, al día pre-tratamiento. En la $2^{\mathrm{a}}$ etapa, se establecieron dos grupos experimentales de 15 animales cada uno, grupo tratado (gT) y grupo control sin tratamiento $(\mathrm{gC})$. Siguiendo la metodología sugerida por la Asociación Mundial para los Avances en Parasitología Veterinaria WAAVP (por sus siglas en inglés the World Association for the Advancement Veterinary Parasitology) ${ }^{(20-22)}$, los animales se seleccionaron al azar y como condición que tuvieran un mínimo de $150 \mathrm{HPG}$. Los rangos presentados en cada grupo fueron de 150 a 2,850 HPG para gC y 150 a 2,150 HPG para gT. Al día cero, al gT se administró IVM al 1\% vía oral a la dosis recomendada de $0.2 \mathrm{mg} / \mathrm{kg}$ de peso vivo ${ }^{(21)}$. Para calcular la dosis individual del fármaco, el peso corporal aproximado se obtuvo mediante el estimador de peso "The Donkey Sanctuary"(23,24). Se tomaron muestras fecales a ambos grupos los días 7 y 14 posttratamiento $^{(21)}$.

\section{Umbrales económicos}

\section{Huevos por gramo de heces (HPG)}

Para conocer el número de HPG se obtuvieron muestras de heces directamente del recto de los burros ${ }^{(21,22)}$. Las muestras se conservaron a $4{ }^{\circ} \mathrm{C}$ hasta ser procesadas mediante la técnica de McMaster modificada ${ }^{(20-22)}$ en el laboratorio de Investigación del Departamento de Parasitología de la FMVZ, UNAM.

\section{Condición corporal (CC)}

La CC se obtuvo a partir de la estimación visual del animal, en una escala del 1 al 5, con incrementos de 0.5, utilizando "Body Condition Score Chart" de The Donkey Sanctuary ${ }^{(23)}$. Identificación de géneros de nematodos gastrointestinales

Se realizaron coprocultivos, para la obtención de la larva infectante $\left(\mathrm{L}_{3}\right)$ mediante la técnica de cultivo descrita por Figueroa $e t a l^{(25)}$ y la técnica de migración de las larvas con el dispositivo Baermann con una incubación de 10 días ${ }^{(25)}$. Posteriormente, se recolectaron las $\mathrm{L}_{3}$ y se lavaron mediante la técnica para la limpieza de larvas de NGI por gradientes de densidad con sacarosa al $40 \%{ }^{(25,26)}$. Finalmente, se identificaron $100 \mathrm{~L}_{3}$ de los cultivos del día pre-tratamiento y de los días 7 y 14 post-tratamiento, respectivamente ${ }^{(25,26)}$. 


\section{Prueba de reducción en el conteo de huevos en heces (FECRT)}

Para determinar el porcentaje de eficacia del fármaco, se realizó la prueba de reducción en el conteo de huevos en heces, FECRT (por sus siglas en inglés Faecal Egg Count Reduction Test) bajo los lineamientos de la WAAVP ${ }^{(21,22,27)}$. A los grupos gT $(\mathrm{n}=15)$ y gC $(\mathrm{n}=15)$, se realizaron pruebas de McMaster de los días 1 pretratamiento, 7 y 14 postratamiento.

\section{Encuestas a los propietarios de los burros}

Se realizó una encuesta exploratoria ${ }^{(28,29)}$ a 25 propietarios de los burros. El cuestionario incluía preguntas acerca de la atención médica dada por los médicos, específicamente la desparasitación, así como el tipo de encierro y alimentación dado a sus animales, con el objetivo de detectar fallas en el manejo y causas que generen RAH. En caso de que el propietario proporcionara el tratamiento complementario al del MVZ, la encuesta consideró cuestiones como los criterios de la frecuencia, procedimientos de aplicación y aplicación de otros antihelmínticos ${ }^{(29)}$.

\section{Análisis estadístico}

Los datos de correlación de CC y HPG, se analizaron con el paquete estadístico R@ ${ }^{(30)}$.Los valores de FECRT se calcularon usando el programa RESO.exe@ ${ }^{(31)}$ de acuerdo con la fórmula: Eficacia $(\%)=(\mathrm{HPG}$ pre- tratamiento $\mathrm{gC}-\mathrm{HPG}$ post-tratamiento gT / HPG pretratamiento $\mathrm{gC}$ ) $\times 100^{(20-22)}$. Se considera susceptible una población parásita a un $\mathrm{AH}$ cuando el porcentaje de eficacia es mayor al $95 \%$ y el límite inferior del intervalo de confianza $95 \%$ es mayor de $90 \%$. Se considera sospechosa a RAH a una población cuando solo uno de los dos criterios se cumple (20-22,31). $^{2}$

\section{Resultados}

\section{Umbrales económicos}

\section{Huevos por gramo de heces (HPG)}

En la $1^{\mathrm{a}}$ etapa, el $98.1 \%(\mathrm{n}=52)$ de las muestras de heces resultaron positivas a huevos tipo estrongílido. El rango de eliminación de HPG en esta etapa va desde los 0 hasta los 3,000 HPG, como se muestra en la Gráfica 1. De acuerdo con la distribución y los valores de la mediana de 350 HPG y el tercer cuartil de 600 HPG obtenidos (Figura 1), se determinó que el umbral económico de los burros en el Altiplano Mexicano es de 600 HPG. 
Figura 1: Distribución de la eliminación de huevos por gramo de heces (HPG), obtenida en burros del altiplano mexicano

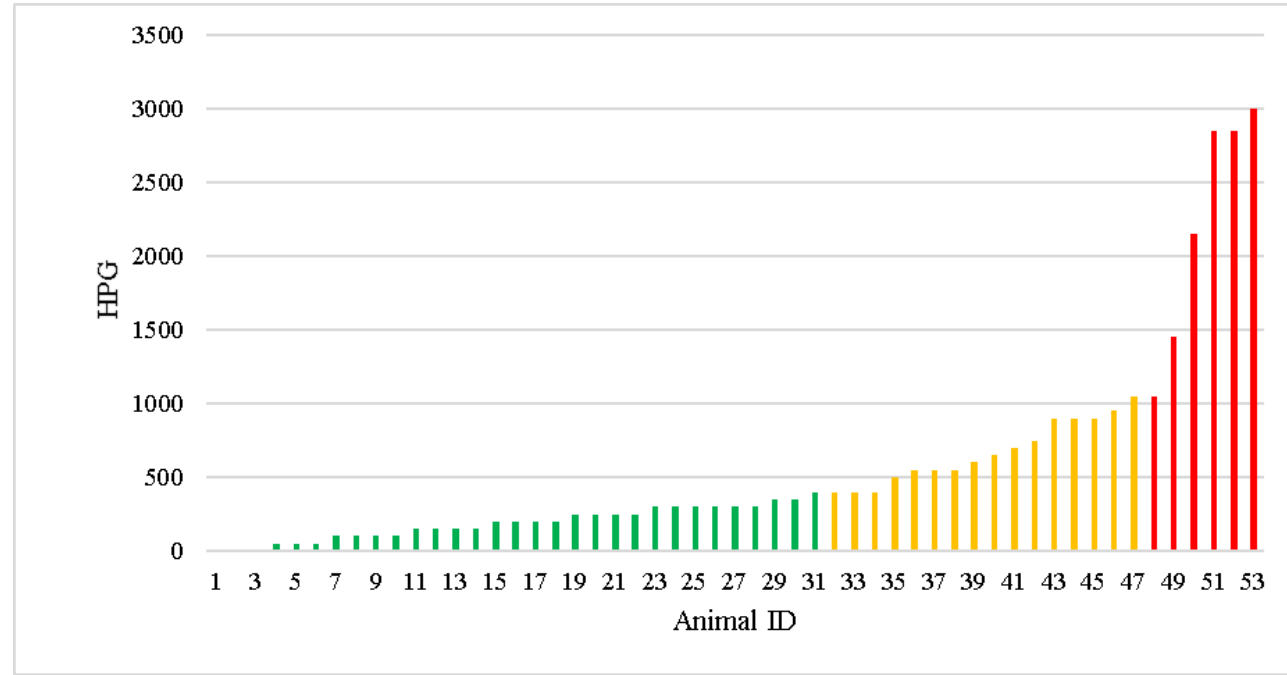

Barras verdes $=$ animales con $<350 \mathrm{HPG}$;

Barras naranjas $=$ animales con 350 a $600 \mathrm{HPG}$;

Barras rojas $=$ animales con $>600 \mathrm{HPG}$.

\section{Condición corporal $(\mathbf{C C})$}

La CC que presentó la mayoría (91\%) de los animales fue aceptable (2.5 a 3.5) y 3 tuvieron valores de 4.5 (sobrepeso) y sólo 2 animales de 1.5 (pobre), según la carta de estimación de condición y peso referida ${ }^{(23)}$. Además, se observó que existe una correlación negativa altamente significativa $(P=0.01)$, por lo que hay evidencia para suponer que, a mayor $\mathrm{CC}$, la descarga de huevos obtenida es menor, con una $P<0.05$ con intervalo de confianza del $95 \%$ (0.07 a 0.56). El coeficiente de determinación fue de 0.1154 (Figura 2). Por lo tanto, por cada aumento en la CC de 0.5, la descarga de huevos se ve reducida $0.33 \%$. Sin embargo, esta disminución en la eliminación de huevos puede ser variable dentro de un rango del 0.07 a $0.56 \%$. 
Figura 2: Correlación entre la eliminación de huevos por gramo de heces (HPG) y la condición corporal (CC) evaluadas en burros del altiplano mexicano

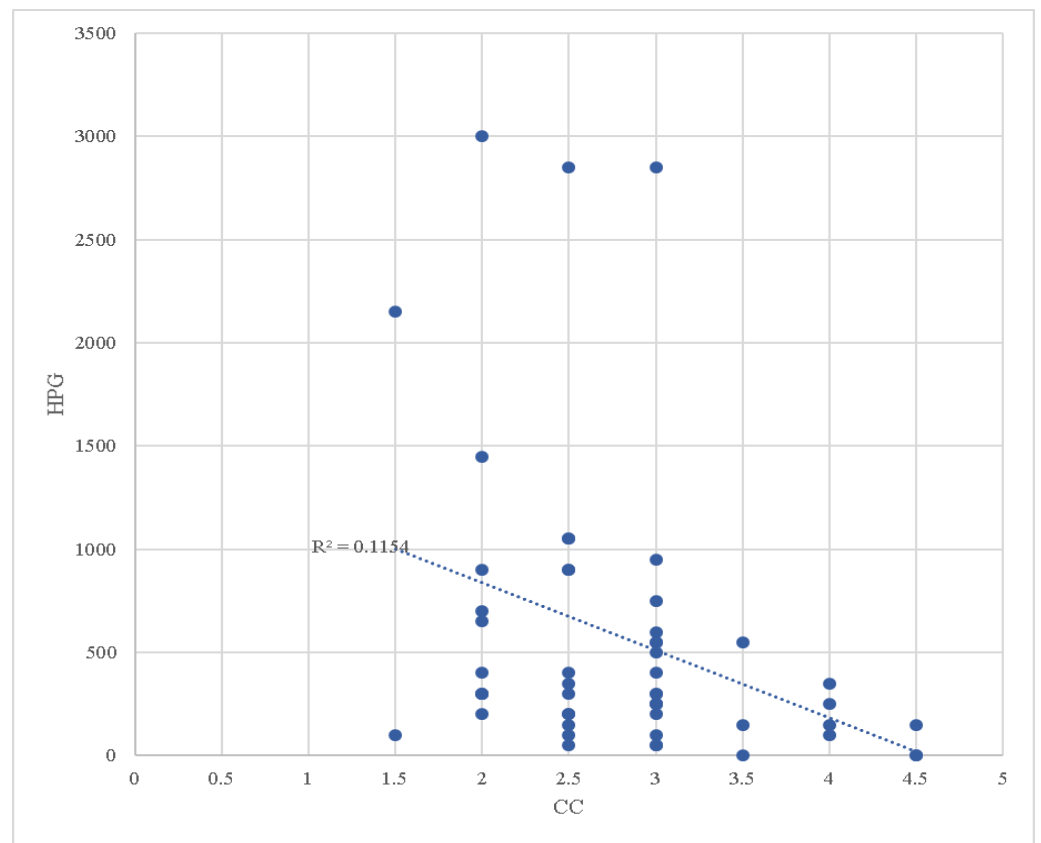

\section{Identificación de géneros de nematodos gastrointestinales}

Fueron identificadas 100 larvas infectantes, donde se observó que el $63 \%$ de éstas corresponden a ciatostómidos (Figura 3). Dentro de los pequeños estrongílidos se encontraron los del género Poteriostomum spp, Gyalocephalus spp y Oesophagodontus spp. La especie con mayor frecuencia de los grandes estrongílidos fue Strongylus edentatus, seguido de S. equinus y $S$. vulgaris.

Figura 3: Distribución de géneros de nematodos gastrointestinales presentes en burros del altiplano mexicano

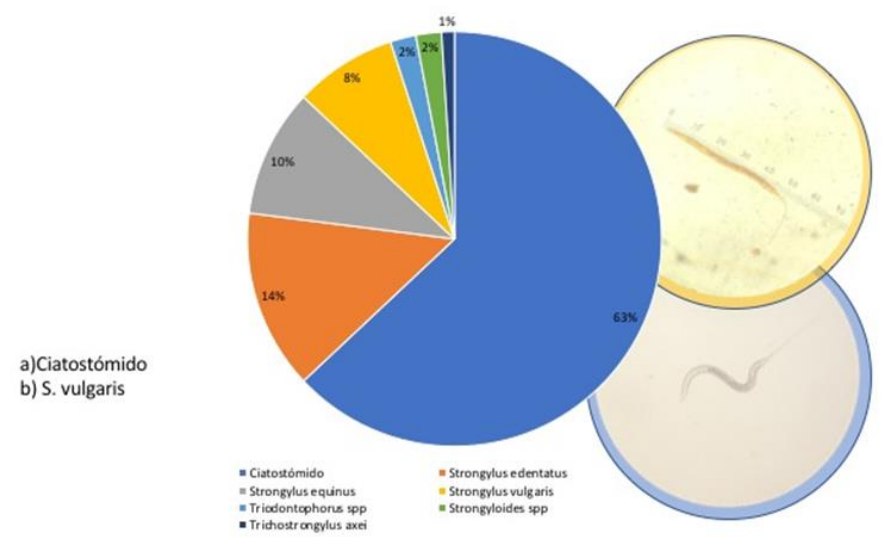




\section{Prueba para determinar la eficacia de la ivermectina FECRT}

En la $2^{\text {a }}$ etapa del experimento, con la prueba FECRT, se obtuvo que los días 7 y 14 posttratamiento en el gT comparado con el gC, la IVM al 1\% administrada vía oral tiene actualmente en esta población específica una eficacia del 99 y $100 \%$ respectivamente, con un intervalo de confianza del $95 \%$, por lo que se considera esta población de parásitos susceptible a la IVM al 1\%. En contraste, el rango de eliminación para el gC fue de 100 a 850 HPG al día 14.

\section{Encuestas a los propietarios de los burros}

La edad promedio de los animales es de 11.05 años, la mayoría machos (79 \%). Solo el $40 \%$ de los animales conviven en corrales, algunos en alojamiento en común en cada comunidad, durante la época de secas, temporada en la que estos no realizan actividades agrícolas cotidianas, aunque el resto indica que cuando éstas son realizadas en las épocas de siembra y cosecha, estos animales en su mayoría son alojados en corrales como encierro nocturno, posterior a la jornada laboral. En el caso de los animales que pastorean, el propietario comenta que es un predio propio, exclusivamente utilizado para la siembra de diversos productos como el maíz y frijol. El $80 \%$ admite utilizar como única estrategia de control parasitario que es proporcionado por el servicio de los médicos voluntarios. El $20 \%$ restante, desconocen el nombre del producto comercial, así como el principio activo y dosifican el producto sin conocer el peso del animal o la dosis. Los propietarios señalan que el tratamiento lo aplican durante la época previa a las lluvias, o hasta dos veces por año (Cuadro 1).

Cuadro 1: Respuestas a la encuesta acerca de las prácticas de desparasitación realizada a propietarios de burros en el altiplano mexicano

\section{Pregunta}

¿Qué manejo da a sus animales

¿Dónde pastorean?

\# animales/corral

Además del servicio MMVVZZ voluntarios, ¿Desparasita a sus animales?

¿Qué producto utiliza?

\begin{tabular}{|l|l|l} 
& \multicolumn{2}{|c|}{$\%$} \\
Pastoreo & 36 & 9 \\
Encierro nocturno & 24 & 6 \\
Corral & 40 & 10 \\
Predio exclusivo & 36 & 9 \\
Predio compartido & 20 & 5 \\
Promedio & NA & 1.3 \\
Si & 20 & 5 \\
No & $\mathbf{8 0}$ & 20 \\
No recuerda & & \\
"Pasta" (no sabe nombre) & 12 & 2
\end{tabular}


¿Verifica la caducidad?

¿A qué dosis aplica el producto?

Vía de Administración

¿Quién aplica el producto?

Pesaje

Frecuencia

¿En qué momento realiza este manejo?

\begin{tabular}{|l|l|l} 
Si & 4 & 1 \\
No & 16 & 4 \\
No sabe/ no recuerda & 12 & 3 \\
Por "cm"/ Todo el & 8 & 2 \\
producto & & \\
Oral & 20 & 5 \\
Intramuscular & 0 & 0 \\
Subcutánea & 0 & 0 \\
No sabe & 0 & 0 \\
MVZ & 8 & 2 \\
Propietario & $\mathbf{1 2}$ & 3 \\
Otro & 4 & 1 \\
Si & 0 & 0 \\
No & $\mathbf{2 0}$ & 5 \\
Cuando el MVZ indica & 0 & 0 \\
Semestral & $\mathbf{1 2}$ & 3 \\
Anual & 8 & 2 \\
Previo empadre & 0 & 0 \\
Previo parto & 0 & 0 \\
Previo a lluvias & $\mathbf{2 0}$ & 5 \\
Después de lluvias & 0 & 0 \\
Otro & 0 & 0
\end{tabular}

\section{Discusión}

En esta especie, en la actualidad, no existe información en el altiplano mexicano donde se conozca: la dinámica poblacional medida en la variación estacional de eliminación de HPG como los realizados anteriormente en Argentina ${ }^{(32)}$, incluyendo la identificación de especies parásitas de la zona; o el umbral económico de HPG propio de la zona ecológica que indique si el animal es candidato o no a la desparasitación, como se ha hecho en otras especies ${ }^{(33,34)}$; o la posible relación entre umbrales, como HPG y CC de los burros en la zona ecológica.

El trabajo de diagnóstico parasitario en burros es pobre, por lo que el establecimiento de umbrales no ha sido prioridad. En trabajos previos ${ }^{(2)}$, se observó una mediana de 600 HPG, y específicamente en una zona ecológica tropical se determinó una mediana de 1,000 HPG, lo que confirma las diferencias que existen entre cada zona. Estas variaciones hablan sobre todo de la epidemiología de los parásitos, de ahí la importancia de definir los umbrales económicos de HPG de manera específica. 
En este estudio, para determinar el criterio de umbral económico de HPG, se obtuvo la distribución donde la mediana fue de $350 \mathrm{HPG}$, lo que significa que de manera rutinaria sea desparasitado al $50 \%$ de los animales. Esto implica un costoso programa de desparasitación innecesario, ya que algunos no necesitan el medicamento. El reto aquí comienza con el llamado fenómeno de la superdispersión en el que varios autores ${ }^{(35,36)}$ coinciden en que sólo el 20 al $25 \%$ de la población animal sufren una parasitosis (por ser grandes eliminadores) y son los probables candidatos al tratamiento. Además, se calculó el valor del tercer cuartil (75\%) el cual fue de 600 HPG, mismo que se tomó como umbral económico de HPG en burros del altiplano mexicano. Esto indicaría que sólo un $25 \%$ de esta población sería candidata a tratamiento, siempre y cuando presentaran otros criterios de umbrales económicos que reflejen una aparente parasitosis. Otro aspecto para evaluar con este estudio de la dispersión de los NGI es que aquellos burros que se ubican en las barras verdes y naranjas representan a la población resistente a los NGI y aquellos animales que están en las barras rojas, pero no presentan signos clínicos y estado de ánimo decaído es la población resiliente a los NGI. Estas dos poblaciones resistentes y resilientes son muy probablemente la importante y rescatable población refugio ante el fenómeno de la RAH, conceptos muy importantes para entender el fenómeno del parasitismo y sus hospederos ${ }^{(37)}$. Considerando también la zona ecológica, resalta el fenómeno de eliminación que se observa en el gC, el cual a los 14 días disminuyó el valor de HPG en un rango de 100 a 850 cuando al pretratamiento fue de 150 a 2,850. Una hipótesis ante esto es el rol de las plantas endémicas con componentes bioactivos contra los NGI, estudiado en otras especies ${ }^{(38)}$ así como el fenómeno de hipobiosis que se discute más adelante.

Los ciatóstomidos han ganado importancia debido al enquistamiento de las larvas en la submucosa intestinal y de las larvas hipobióticas ${ }^{(39-41)}$. A su vez, es sabido que en países donde en el periodo de otoño e invierno es marcado, momento donde fue realizado este estudio, las larvas que podrían encontrarse enquistadas en la mucosa intestinal son indetectables ${ }^{(21,39)}$, por lo que el conocer la dinámica de población parasitaria anual podría dar un mejor panorama de las nematodosis existentes y a su vez, desarrollar estrategias para su tratamiento.

Es importante mencionar que la CC obtenida en el $91 \%$ de los burros tiene valores aceptables de 2.5 a 3.5, lo que indica que los animales a pesar de sus condiciones medio ambientales expresan la rusticidad que los caracteriza y hacen frente a las parasitosis ${ }^{(3)}$, fenómeno que ayuda a reafirmar por qué no todos los burros necesitan un tratamiento bajo esquemas fijos. Por otra parte, cuando se hizo la correlación entre la CC y el valor de HPG, se observó que existe una aparente reducción del $0.33 \%$ de HPG, conforme el aumento de 0.5 en CC. Este estudio, definió que los HPG, la CC y su estrecha relación, son importantes umbrales en el manejo de las nematodosis gastrointestinales en burros. Es importante señalar que, aunque existen estudios donde no se observa una relación entre la CC y la carga parasitaria en burros $^{(2,3,23)}$, Yoseph et $a l^{(40)}$ sugieren la medición de este umbral económico, siempre y 
cuando se cumpla con una alimentación basada en forraje de buena calidad y un adecuado manejo del tratamiento antihelmíntico.

Dado que las parasitosis son unas de las enfermedades que pasan desapercibidas por el propietario, varios autores ${ }^{(41)}$ mencionan la importancia de que se restablezca la asociación entre médico y productor/propietario para el diseño de estrategias de manejo y control parasitario, aunado con la creciente preocupación por la RAH en México, debido al uso constante e indiscriminado de los AH. Esto sucede principalmente con la IVM.

En este estudio se comprobó que la efectividad de la IVM fue del $100 \%$. Sin embargo, existen estudios ${ }^{(42,43)}$ en los que se menciona, que debido a que las pruebas para la efectividad de $\mathrm{AH}$ en équidos aún no se han estandarizado del todo, es posible que exista un margen donde la validación de una población resistente requiera de pruebas complementarias. También otros autores ${ }^{(44)}$ reportan que es de importancia el desarrollo de pruebas comparativas como el LMIT (test de la inhibición de migración larvaria) en la que encontró que es posible conocer la sensibilidad a la IVM de la población de ciatostómidos presentes en équidos. Complementario a este estudio, se sugiere la evaluación del ERP (el periodo de reaparición de huevos por sus siglas en inglés) después del tratamiento, debido a que se ha observado que, aunque el FECRT indique que la población parásita es sensible en un $100 \%$ al AH evaluado, este umbral puede exponer un proceso de resistencia latente. En équidos, el ERP que se observó es de 8 a 13 semanas para IVM. En burros de The Donkey Sanctuary en Reino Unido, este $E R P$ en animales que han sido poco expuestos al fármaco, es de 6 semanas ${ }^{(44)}$ y probablemente se ha visto reducido debido al desarrollo de especímenes "juveniles" que no fueron eliminados durante el tratamiento ${ }^{(45)}$. La poca efectividad del fármaco contra larvas hipobióticas podría ser un detonante de RAH, debido a la exposición constante de éstas a la IVM, por lo que se ve aumentada la presión de selección del parásito, manifestándose en un corto $E R P$, y por lo tanto especies resistentes en la siguiente generación ${ }^{(44)}$; sin embargo, más estudios son necesarios para corroborar esto.

No se puede hablar de la efectividad de un fármaco o de RAH, si no se evalúa de manera paralela las prácticas que propietarios o veterinarios realizan con respecto a la actividad de desparasitación. En este estudio se detectaron probables causas de RAH y posibles fallas en la desparasitación a través de encuestas.

Se daba tratamiento a toda la población de burros, incluso aquellos aparentemente sanos. Esto aumenta la presión de selección de los parásitos, haciendo que las generaciones siguientes sean resistentes y disminuyendo la existencia de hospederos refugio dentro de la población, por lo que la reserva de un valioso recurso, como lo es la IVM, para animales que realmente requieren tratamiento, evita la generación de nuevas especies no sensibles a ésta. Se dosificaba sin conocer el peso corporal exacto, así como un tratamiento sistemático o supresivo (p.ej. dos veces por año) de la misma AH. En este caso, los riesgos de estas 
acciones representan que los parásitos adquieran la RAH sin una eficiente eliminación o control y la hereden a su descendencia. Un ejemplo claro, es la reducida acción de la IVM contra larvas hipobióticas, en donde se ve aumentada la presión de selección en el parásito. El desconocimiento del propietario de los productos aplicados, así como de dosis correctas, objetivo del manejo y la correcta aplicación de este, ya que, como se menciona anteriormente, pueden ocurrir tratamientos inadvertidos y la subdosificación/ sobredosificación del fármaco.

Por lo anterior, la implementación de nuevas herramientas de control parasitario, que tengan como objetivo disminuir la presión de selección de los parásitos a distintos $\mathrm{AH}$, se ha convertido en una de las principales necesidades del manejo parasitario ${ }^{(41)}$. Respondiendo a esta necesidad, surge la utilización de distintas herramientas que finalmente conforman un manejo y control que las integre. El CIP pretende retardar el aumento de poblaciones parásitas con mayor proporción de individuos genéticamente resistentes a uno o más $\mathrm{AH}^{(46)}$. El esquema propone integrar distintos principios basados en la dinámica poblacional parásita de un hato o establo conocido. Dentro de este esquema se encuentra la $\operatorname{DSD}^{(16)}$, la cual se percibe como viable en esta región.

\section{Conclusiones e implicaciones}

Si bien el desarrollo de resistencia a la IVM aún no está presente, lo observado demuestra que estas comunidades se encuentran en un punto crítico, por lo que la corrección de las fallas en el tratamiento antihelmíntico y la prevención de causas probables de RAH pueden ayudar a los médicos, en conjunto con los propietarios, a cuidar un recurso único como lo es este AH a través de la utilización adecuada y racional de nuevas moléculas disponibles en el mercado, la evaluación y obtención de umbrales propios junto con el desarrollo de herramientas para el establecimiento de estos, dejando como precedente la concientización del uso correcto de los AH y, en consecuencia, el mejoramiento en la calidad del bienestar animal de los burros.

\section{Agradecimientos}

Al MVZ. Omar Prado Ortiz por la valiosa ayuda técnica recibida durante el desarrollo de la investigación.

\section{Literatura citada:}

1. The Donkey Sanctuary. Svendsen ED [compilador]. Manual Profesional del Burro. Reino Unido: Whittet Books; 1997.

2. Burden F, Du Toit N, Hernández Gil M, Prado Ortiz O, Trawford AF. Selected health and management issues facing working donkeys presented for veterinary treatment in rural 
Mexico: some possible risk factors and potential intervention strategies. Trop Anim Health Prod 2010;42:597-605.

3. Burden F, Thiemann A. Donkeys Are Different. J Eq Vet Sci 2915; 35:376-382.

4. Corning S. Equine cyathostomins: a review of biology, clinical significance and therapy. Parasites \& Vectors 2009;2(suppl 2):S1.

5. Canever RJ, Braga PRC, Boeckh A, Gycajuck M, Bier D, Molento MB. Lack of Cyathostomin sp. reduction after anthelmintic treatment in horses in Brazil. Vet Parasitol 2013;194:35-39.

6. Taylor MA, Coop RL, Wall RL. Veterinary Parasitology. 4a ed. Oxford, Reino Unido: Wiley-Blackwell; 2015.

7. ITIS. Integrated Taxonomic Information System. Encyclopedia of Life (EOL). https://www.itis.gov/servlet/SingleRpt/SingleRpt?search_topic=TSN\&search _value=63957\#null. Consultado 1 Mar, 2017.

8. Matthews J, Burden F. Common helminth infections of donkeys and their control in temperate regions. Eq Vet Ed 2013;25:461-467.

9. Grosenbaugh DA, Reinemeyer CR, Figueiredo MD. Pharmacology and therapeutics in Donkeys. Eq Vet Ed 2011;23:523-530.

10. Vercruysse J, Claerebout E. Treatment vs non-treatment of helmint infections in cattle: defining the threshold. Vet Parasitol 2001;98:195-214.

11. Kaplan R, Vidyashankar A. An inconvenient truth: Global worming and anthelmintic resistance. Vet Parasitol 2002;186(1-2):70-78.

12. Nari A, Eddi C. Control Integrado de las parasitosis. Reunión de especialistas en parasitología Veterinaria de Argentina, Brasil, Chile, Paraguay y Uruguay. Tandil, Argentina; 2002.

13. Torres Acosta JFJ, Hoste H. Alternative or improved methods to limit gastro-intestinal parasitism in grazing sheep and goats. Small Ruminant Res 2008;77:159-173.

14. González Garduño R, Torres Hernández G, López Arellano ME, Mendoza-de-Gives P. Resistencia antihelmíntica de nematodos parásitos en ovinos. Rev Geo Ag 2012; 48(49): 63-74.

15. Rosado-Aguilar JA, Flota-Burgos GJ, Rojas-Becerril R, Trinidad-Martínez I. Frequency and ivermectin resistance of gastrointestinal nematodes in equine farms from Mexico [summary].13th Int Cong Parasitol. Mexico. 2014. 
16. Van Wik J, Hoste H, Kaplan RM, Besier RB. Targeted selective treatment form worm management-How do we sell rational programs to farmers. Vet Parasitol 2006;139:336346.

17. Berk Z, Laurenson YCSM, Forbes AB, Kyriazakis I. Modelling the consequences of targeted selective treatment strategies on performance and emergence of anthelmintic resistance amongst grazing calves. IJPl: Drugs and Drugs Resistance 2016;6:258-271.

18. Martínez Ortiz-de-Montellano C, Torres Acosta JFJ. Control integrado de los nematodos gastrointestinales en rumiantes. Consejo Técnico Nacional de Sanidad Animal. Tuxtla Gutiérrez, Chiapas. 2011.

19. Vineer HR, Vande Velde F, Bull K, Claerebout E, Morgan ER. Attitudes towards worm egg counts and targeted selective treatment against equine cyathostomins. Prev Vet Med 2017;144:66-74.

20. Duncan JL, About EM, Arundel JH, Eyseker M, Lei TR, Krecek RC et al. World association for the advancement of veterinary parasitology (WAAVP). Second ed. Guidelines for evaluating the efficacy of equine anthelmintics. Vet Parasitol 2002;103(1-2):1-18.

21. Wood IB, Amaral NK, Bairden K, Duncan JL, Kassai T, Malone JB et al. World Association for the advancement of veterinary parasitology (WAAVP). Second edition of guidelines for evaluating the efficacy of anthelmintics in ruminants (bovine, ovine, caprine). Vet Parasitol 1995;58:181-213.

22. Geurden T, van Doorn D, Claerebout E, Kooyman F, De Keermaecker S, Vercruysse J et al. Decreased strongyle egg re-appearance period after treatment with ivermectin and moxidectin in horses in Belgium, Italy and The Netherlands. Vet Parasitol 2014;204(34):291-296.

23. The Donkey Sanctuary. Condition scoring and weight estimation. Devon, Reino Unido: The Donkey Sanctuary. 2013.

24. The Donkey Sanctuary. Condition scoring and weight estimation. Devon, Reino Unido: The Donkey Sanctuary 2014; https://www.thedonkeysanctuary.org.uk/sites/sanctuary/files/document/1421423234830-donkey_health_and_welfare.pdf. Consultado Mar 30, 2017.

25. Figueroa JA et al. Examen coproparasitoscópico. En: Rodríguez Vivas RI, editor. Técnicas para el diagnóstico de parásitos con importancia en salud pública y veterinaria. $1^{\mathrm{a}}$. ed. México: AMPAVE. Consejo Técnico Consultivo Nacional de Sanidad Animal. 2015:101-118. 
26. Zajac MA, Conboy GA. Veterinary Clinical Parasitology. 8a ed. USA: Am Assoc Vet Parasitol. 2012.

27. Cabaret J, Berrag B. Faecal egg count reduction test for assessing anthelmintic efficacy: average versus individually based estimations. Vet Parasitol 2004;121:105-113

28. López Romo H. La metodología de encuesta en Galindo Caceres LJ, coordinador. Técnicas de investigación en sociedad, cultura y comunicación. México: Logman; 1998:83-93.

29. Alegría López MA, Rodríguez Vivas RI, Torres Acosta JFJ, Ojeda Chi MM, Rosado Aguilar JA. Use of Ivermectin as endoparasitide in tropical cattle herds generates resistance in gastrointestinal nematodes and the tick Rhipicephalus microplus (Acari: Ixodidae). J Med Entomol 2015;52:214-221.

30. R Development Core Team. R: A language and environment for statistical computing. R Foundation for Statistical Computing, Vienna, Austria; 2018. ISBN 3-900051-07-0, URL http://www.R-project.org.

31. Rodríguez Vivas RI [editor]. Técnicas para el diagnóstico de parásitos con importancia en salud pública y veterinaria. 1a Ed. México: AMPAVE / Consejo Técnico Consultivo Nacional de Sanidad Animal. 2015:355-403.

32. Fusé L, Saumell CA, Iglesias L. Variación estacional del parasitismo interno en equinos: Fenómeno de hipobiosis de los pequeños estrongílidos (Cyathostominae) en Tandil, Buenos Aires, Argentina. Rev Med Vet 2013;94(3):62-72.

33. Martinez Ortiz-de-Montellano C, Cervantes Morali J, Figueroa Castillo JA, Torres Acosta JFJ. Distribution of gastrointestinal nematodes egg counts in sheep from Mexico Center south and its implications of parasitic integrated control. Int Conf World Assoc Advanc Vet Parasitol. Kuala Lumpur, Malasia; 2017.

34. Martínez-de-Castro Dubernard A, Dominguez Hernández Y, Quiroz Rocha G, Saldaña Hernandez $\mathrm{N}$, Ojeda Robertos $\mathrm{N}$ et al. Importance of egg per gram treshold determination in goats from Mexican plateau: To deworm or not to deworm?. Int Conf World Assoc Advanc Vet Parasitol. Kuala Lumpur, Malasia; 2017.

35. Sréter T, Molnár V, Kassai T. The distribution of nematode egg counts and larval counts in grazing sheep and their implications for parasite control. Int J Parasitol 1994; 24(1):103-108.

36. Hoste H, Le Frieleux Y, Pommaret A. Distribution and repeatability of feacal egg counts and blood parameters in dairy goats naturally infected with gastrointestinal nematodes. Res in Vet Sci 2011;70(1):57-60. 
37. Van-Houtert MFJ, Sykes AR. Implications of nutriction for the ability of ruminants to withstand gastrointestinal nematode infections. Int J Parasitol 1996;26(11):1151-1167.

38. Peachey LE, Pinchbeck GL, Matthews JB, Burden FA, Mulugueta G, Scantlebury CE et $a l$. An evidence-based approach to the evaluation of ethnoveterinary medicines against strongyle nematodes of equids. Vet Parasitol 2015;210:40-52.

39. Zachary JF. Pathologic basis of Veterinary diseases. $6^{\text {th }}$ ed. USA; 20017.

40. Yoseph S, Smith DG, Teklu F, Firew T, Betere Y. Seasonal variation in the parasite burden and body condition of working donkeys in East Shewa and West Shewa Regions of Ethiopia. Trop Anim Health Prod 2005;37(1):35-45.

41. Statford CH, McGorum BC, Pickles KJ, Matthews JB. An update on cyathostomins: Anthelmintic resistance and diagnostic tools. Eq Vet J 2011;43:133-139.

42. Molento MB, Nielsen K, Kaplan RM. Resistance to avermectin/milbemycin anthelmintics in equine cyathostomins - Current situation. Vet Parasitol 2012;185:1624.

43. Mejía ME, Fernández Igartúa BM, Schmidt EE, Cabaret J. Multispecies and multiple anthelmintic resistance on cattle nematodes in a farm in Argentina: the beginning of high resistance?. Vet Res 2003;34:461-467.

44. McArthur CL, Handel IG, Robison A, Hodgkinson JE, Bronsvoort B, Burden F. et al. Development of the larval migration inhibition test for comparative analysis of ivermectin sensitivity in cyathostomin populations. Vet Parasitol 2015;212:292-298.

45. Lyons ET, Tolliver SC, Collins SS. Probable reason why small strongyle EPG counts returning "early" after ivermectin treatment of horses on a farm in Central Kentucky. Parasitol Res 2009;104(3):569-574.

46. Waller PJ, Echevarría F, Eddi C, Maciel S, Nari A, Hansen JW. The prevalence of anthelmintic resistance in nematodes parasites of sheep Southern Latin America: General overview. Vet Parasitol 1996;62:181-187. 